\title{
Ancylostomiasis due to Ancylostoma Braziliense
}

National Cancer Institute

\section{Source}

National Cancer Institute. Ancylostomiasis due to Ancylostoma Braziliense. NCI

Thesaurus. Code C35175.

Infection by Ancylostoma braziliense. 